\title{
HIDROCELE REACTIVO TRAS PALOMO LAPAROSCÓPICO EN EL VARICOCELE PEDIÁTRICO
}

\author{
Roberto Méndez-Gallart', Adolfo Bautista Casasnovas, Elina Estévez Martínez, Pablo Rodríguez- \\ Barca, Pedro Taboada Santomil, Azucena Armas, Jose Pradillos, Lizet Rivera y Ramiro Varela \\ Cives.
}

Servicio de Cirugía Pediátrica. Hospital Clínico Universitario de Santiago. Santiago de Compostela. La Coruña. España.

\begin{abstract}
Resumen.- OBJETIVO: Evaluar la incidencia de hidrocele reactivo tras la técnica de Palomo laparoscópico en pacientes menores de 19 años en nuestro centro.

MÉTODOS: Entre los años 1997 y 2009, 180 varones menores de 19 años diagnosticados de varicocele fueron incluidos en el estudio. Las variables registradas fueron edad, sintomatología, grado de Varicocele según la clasificación de Dubin-Amelar, tamaño testicular, estancia hospitalaria, complicaciones, recurrencia y formación de hidrocele tras la intervención. El seguimiento medio fue de 5 años (6 meses a 9 años).
\end{abstract}

RESULTADOS: La edad al diagnóstico fue de 9 a 19 años. 177 fueron varicoceles izquierdos (98\%) y 4 ca-

\section{CORRESPONDENCIA}

Roberto Méndez-Gallart

Servicio de Cirugía Pediátrica

Hospital Clínico Universitario de Santiago

Travesía Choupana s/n

15706 Santiago de Compostela

La Coruña (España)

roberto.mendez.gallart@sergas.es

Aceptado para publicar: 21 de mayo 2010. sos fueron bilaterales. Todos tenían varicocele grados II - III y atrofia testicular un 45\%; El $8.1 \%$ referían dolor testicular. Todos los pacientes se operaron mediante un procedimiento de Palomo laparoscópico. El tiempo medio de duración de la intervención fue de 38 minutos. Las últimas 63 cirugías se realizaron solo con 2 trocares y Ligasure para sellar los vasos, logrando disminuir el tiempo quirúrgico a 22 minutos. La estancia hospitalaria media fue de 31 h. 23 pacientes desarrollaron hidrocele reactivo (13\%); 11 de los cuales precisaron una hidrocelectomía (procedimiento de Winkelman-Lord) como mínimo un año después de la primera intervención laparoscópica. De los restantes, en 2 casos se resolvió espontáneamente y 10 casos permanecen estables con un hidrocele leve tras 4 años de seguimiento.

CONCLUSIONES: Según nuestro estudio, la corrección laparoscópica del varicocele es una técnica segura, eficaz e idónea para su empleo en pacientes pediátricos y adolescentes. La complicación más indeseable y frecuente es el hidrocele reactivo, que a veces puede aparecer mas de un año después de la intervención. Debido a este hecho, la incidencia de hidrocele descrita en los estudios puede ser inferior a la real.

Palabras clave: Varicocele. Palomo. Varicocelectomía laparoscópica. Hidrocele reactivo.

Summary.- OBJECTIVES: To evaluate the incidence of hydrocele following laparoscopic Palomo varicocele ligation in pediatric and adolescents in our institution.

METHODS: Between 1997 and 2009, 180 boys diagnosed as having varicocele who underwent Palomo laparoscopic ligation were evaluated retrospective- 
1y. Outcome variables recorded for analysis were age at presentation, symptoms, varicocele grade (Dubin-Amelar classification), testicular atrophy, length of hospital stay, perioperative complications, recurrence and hydrocele formation after surgery. Mean follow up was 5.8 years (6 months- 9 years).

RESULTS: Age at diagnosis ranged between 9 and 19 years. Mean age at operation was $14.1 \pm 1.8$ years. There were 177 left-side varicoceles (98\%) and four cases were bilateral. Testicular atrophy was noted in 45. Mean operative time was $38 \mathrm{~min}$. The last 63 surgeries were performed on a two-trocar basis with Ligasure vascular sealing device and operative time decreased significantly to $22 \mathrm{~min}$. Median hospital stay was $31 \mathrm{~h}$. Twenty-three patients developed hydrocele (13\%); 11 of these underwent Winkelman-Lord's hydrocelectomy at least 7 year after Palomo (9\% of totall. Of the remaining 12, two resolved spontaneously and 10 were stable at mean 4-year follow up.

CONCLUSIONS: Laparoscopic Palomo procedure is a safe successful method to correct varicoceles in pediatric and adolescent males. Reactive hydrocele formation following laparoscopic varicocelectomy is a worrisome problem particularly in cases with longer follow-up.

Keywords: Varicocele. Palomo. Laparoscopic varicocelectomy. Reactive Hydrocele.

\section{INTRODUCCIÓN}

El varicocele es una patología muy frecuente en niños mayores y adolescentes. Su incidencia aumenta de forma progresiva desde los 10-12 años hasta la edad adulta, llegando a afectar hasta al 15\% de los adolescentes (1-3). La dilatación de las venas espermáticas del plexo pampiniforme, es secundaria a un fallo en la competencia valvular del drenaje venoso del testículo y afecta predominantemente al testículo izquierdo. El varicocele puede dar lugar a atrofia testicular incluso en pacientes prepuberales y es la causa más común de infertilidad en adultos jóvenes (4). La corrección quirúrgica se recomienda en aquellos casos clínicamente evidentes y con ecografía doppler sugestiva. El objetivo del tratamiento quirúrgico es revertir el daño sobre el testículo y recuperar el potencial de fertilidad del mismo mejorando la calidad en la producción de espermatozoides (5). Entre las técnicas quirúrgicas descritas para su tratamiento, las más empleadas son el abordaje retroperitoneal abierto según Técnica de Palomo, el abordaje inguinal descrito por Ivanissevich, la escleroterapia anterógrada, la embolizacion retrógrada endovascular, y la ligadura espermática laparoscó- pica y retroperitoneoscópica (6-11). Desde que en 1988 fuese descrita por primera vez, la ligadura alta de las venas espermáticas por laparoscopia (Técnica de Palomo laparoscópica) se ha convertido en una técnica preferente para la corrección del varicocele en pacientes pediátricos y adolescentes (12). Independientemente de la técnica quirúrgica elegida, la aparición de hidrocele reactivo es la complicación mas frecuente tras la corrección del varicocele. Los diferentes autores han registrado tasa de hidrocele incluso próximas al 30\% (13-15).

El objetivo de nuestro trabajo es el de evaluar la aparición de hidrocele reactivo en nuestra serie tras el tratamiento del varicocele en niños mayores y adolescentes mediante la ligadura espermática alta (técnica de Palomo) por vía laparoscópica.

\section{MATERIAL Y MÉTODO}

Revisamos retrospectivamente los historiales clínicos de 180 pacientes diagnosticados de varicocele tratados mediante Técnica de Palomo laparoscópica por el Servicio de Cirugía Pediátrica del Complexo Hospitalario Universitario de Santiago desde 1997 a 2009. Todos los pacientes fueron diagnosticados clínicamente y con ecografía doppler color, que visualizó el flujo retrógrado en las venas espermáticas tras la maniobra de Valsalva. Empleamos la clasificación clínica en tres grados de Dubin y Amelar para catalogar a los pacientes (16). Medimos el tamaño de los testes con un orquidómetro de Prader. Únicamente se indicó la intervención en aquellos casos con varicocele grado III o bien con grado II en los que la disminución de tamaño testicular era evidente o el paciente refería molestias escrotales. Ninguno de nuestros pacientes acudió a consulta por problemas de infertilidad.

El procedimiento laparoscópico ya ha sido descrito previamente (17). Tras acceder a la cavidad con técnica abierta a través del ombligo, se estableció el nivel máximo de $\mathrm{CO} 2$ en $14 \mathrm{mmHg}$. Empleamos otro trocar de trabajo de $5 \mathrm{~mm}$ en cuadrante inferior derecho. Una vez visualizados los vasos espermáticos accedemos al retroperitoneo con una incisión de unos $2 \mathrm{cms}$. Tras disecar los vasos en bloque (incluyendo la arteria) y separarlos del psoas, los sellamos empleando un sistema de coagulación

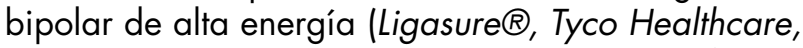
Boulder, Colorado, USA). Los primeros casos fueron ligados con endo-clips, pero modificamos la técnica para minimizar el tiempo quirúrgico. No cerramos el orificio practicado en el peritoneo. Los orificios de los trocares los cerramos con sutura absorbible y Dermabond. En el postoperatorio los pacientes reciben 
propacetamol IV y al día siguiente de la cirugía son dados de alta.

En todos los casos recogimos datos acerca de la edad, presentación clínica, hallazgos ecográficos, tamaño testicular, grado del varicocele, estancia hospitalaria, complicaciones, recurrencia y sobre todo, aparición de hidrocele reactivo. El seguimiento medio fue de 5.8 años. Se mantuvo control en consultas 3 meses después de la intervención y cada 6 meses hasta un mínimo de 2 años del postoperatorio, vigilando especialmente la aparición tardía de atrofia testicular o hidrocele reactivo.

Se realizó un estudio descriptivo de todas las variables incluidas en el trabajo. Para las Varia-bles cuantitativas se utilizó la t de Student o el test $U$ de Mann-Whitney. Para la asociación de variables cualitativas se utilizó el chi-cuadrado ( $\chi 2)$ de Pearson. El nivel de significación estadística esta-blecido en todos los análisis fue de $p<0.05$. El análisis de los datos se llevó a cabo con el programa estadístico SPSS $尺 12.0$.

\section{RESULTADOS}

Analizamos un total de 180 pacientes intervenidos por técnica de Palomo laparoscópico durante un período de 12 años (1997-2009). La edad media de los pacientes al diagnóstico fue de 14.8 años (rango 9-19 años). El 97.9\% de nuestros casos fueron varicoceles izquierdos, mientras que sólo 4 pacientes presentaban afectación bilateral. El 70\% de nuestra serie había sido catalogado de varicocele grado III en la exploración clínica. La disminución del tamaño testicular era evidente en el $45 \%$ de los pacientes. Sólo 14 pacientes relataron molestias testiculares como presentación clínica.

El tiempo medio de intervención fue de 38 min (25-82 min). Las últimos 63 cirugías se realizaron con sólo 2 trocares y Ligasure ${ }^{\circledR}$ para coagulación y sellado de los vasos espermáticos y el tiempo disminuyó a una media de $22 \mathrm{~min}$ (16-32 min) $p<0.05$. La estancia media en el hospital fue de 31 horas (rango de 1 a 7 días). Sólo registramos 2 conversiones durante los primeros pacientes en lo que asumimos como curva de aprendizaje. En un paciente se produjo un sangrado importante por una lesión inadvertida de un vaso epigástrico con un trocar y precisó transfusión. 2 adolescentes refirieron un importante dolor en el hombro derecho durante las primeras 24 horas postoperatorias.

23 pacientes fueron diagnosticados de hidrocele reactivo durante el seguimiento (13\%). Si bien el grupo en el que se empleo Ligasure para sellar los vasos mostró una menor incidencia, ésta no fue estadísticamente significativa $(p=0.23)$. Sólo en 11 pacientes se realizó, debido al tamaño del hidrocele, una corrección quirúrgica mediante la técnica de Winkelman-Lord después de la ligadura laparoscópica ( $9 \%$ del total de casos). De los restantes 12 pacientes, 10 permanecen estables con un tamaño discreto del hidrocele y en 2 casos éste se ha resuelto (seguimiento medio de 4 años en estos pacientes). El diagnóstico de hidrocele reactivo se realizó como media 6 meses después de la intervención. 5 pacientes presentaron el hidrocele 2 años después de la ligadura laparoscópica. Pero todos los casos que precisaron corrección quirúrgica mediante plicatura de la tunica vaginalis presentaban ya un hidrocele aparente a los 3 meses de la intervención. La edad superior a los 15 años ha sido un factor pronóstico para el desarrollo de hidrocele post ligadura laparoscópica $(p<0.05)$. Los pacientes menores de 15 años tienen pocas probabilidades en nuestra experiencia de desarrollar un hidrocele clínicamente significativo tras la técnica de Palomo laparoscópica. El grado de hidrocele no mostró una correlación estadísticamente significativa con la aparición de hidrocele reactivo. El seguimiento medio de todos los pacientes fue de $4.8 \pm 2.5$ años.

La ecografía doppler color realizada para el seguimiento de los pacientes evidenció una resolución del varicocele en el $98.5 \%$ y una persistencia del mismo en 3 casos. Sólo uno de estos 3 casos fue reintervenido (de nuevo por la Técnica de palomo) debido a la existencia de un alto flujo retrógrado en el doppler. Los restantes 2 pacientes están siendo observados debido al bajo flujo mostrado por el plexo venoso en el doppler. No hemos registrado casos de atrofia testicular tras la ligadura laparoscópica, sino que por el contrario, el $63 \%$ de los pacientes mostraron aumento del tamaño del testículo ipsilateral a los dos años de seguimiento.

\section{DISCUSIÓN}

La técnica ideal indicada para la corrección del varicocele en pacientes pediátricos y adolescentes es todavía objeto de controversia. La técnica perfecta sería aquella que tuviese una mínima tasa de recurrencia con la menor incidencia de hidrocele reactivo. La mayoría de los procedimientos que implican baja tasa de recurrencias suelen conllevar alto índice de hidrocele reactivo. Este hecho se cree debido a que los intentos de preservar intactos los linfáticos muchas veces implica la persistencia de pequeñas venas espermáticas que pasan inadvertidas durante el procedimiento y que suponen una recurrencia a 
medio plazo (18). Es necesario que la facilidad de la técnica y el bajo índice de recidivas del varicocele se acompañen de una menor tasa de hidrocele, pero conjugar estos tres factores no es sencillo y ninguna técnica ha superado a las otras a la hora de combinar estos 3 requisitos $(13,14,15,19,20)$.

La escleroterapia anterógrada tiene unas tasas de recidiva de hasta el $18 \%$. La embolizacion selectiva de las venas testiculares es una técnica invasiva que implica un alto nivel de radiación durante un tempo medio de 3 horas que puede durar el procedimiento. El abordaje subinguinal microquirúrgico es el que menor índice de recidivas implica pero el porcentaje de fracasos se eleva hasta el 15\% (21).

El abordaje inguinal descrito por Ivanissevich tiene una tasa de recidivas del $16 \%$ mientras que el abordaje retroperitoneal alto mediante técnica abierta (Palomo) es la intervención que menos índice de recurrencias se han descrito (22). Claramente la técnica de Palomo laparoscópica ha demostrado su facilidad de aprendizaje y su muy bajo índice de recidivas, pero estos datos no se han acompañado de una menor tasa de hidrocele reactivo; antes bien ésta se mantiene estable en un $12-20 \%$ (23). La ligadura inguinal alta en bloque de los vasos espermáticos implica una casi segura transección de los linfáticos eferentes del escroto ipsilateral. Los intentos de preservar estos vasos linfáticos íntimamente adheridos a las venas y arteria espermáticas durante la disección laparoscópica suponen un gran aumento de tiempo quirúrgico, una dificultad elevada de la técnica y un alto riesgo de recidivas por culpa de la preservación inadvertida de pequeños vasos espermáticos que se dejan sin sellar (18).

La técnica de Palomo (ligadura alta de los vasos espermáticos a nivel retroperitoneal) por vía abierta ha sido asociada clásicamente a unos índices elevados de aparición de hidrocele reactivo (próximos al $15-20 \%)$. Por el contrario, las técnicas que implican la preservación de linfáticos han referido cifras de hidrocele reactivo incluso inferiores al 5\% (24-27).

Es cierto que las amplias diferencias registradas entre tasas de aparición de hidrocele reactivo en series cortas y con seguimientos no demasiado prolongados hacen que sacar conclusiones sobre la técnica más idónea sea complicado.

No debemos menospreciar la relevancia del hidrocele reactivo ya que además de las molestias que puede producir el incremento de volumen escrotal especialmente en adolescentes, en teoría también puede alterar el mecanismo de regulación de tem- peratura del teste con las implicaciones que este hecho tendría sobre la fertilidad. Pese a ello seguimos manteniendo que es preferible obtener unas tasas de hidrocele reactivo próximas al $15 \%$ con mínimas tasas de recidiva (inferiores al $3 \%$ ) ya que los intentos de minimizar la aparición de hidrocele tras varicocelectomía han aumentado los índices de recurrencia incluso hasta un $10 \%$ (13).

Es posible que el varicocele pediátrico sea una entidad diferente del varicocele de pacientes adultos como han confirmado otros autores. En nuestra serie hemos comprobado grandes diferencias entre el grupo de pacientes adolescentes y el grupo de menores de 15 años en cuanto a resultados y aparición de hidrocele postoperatorio. Quizás el planteamiento quirúrgico laparoscópico deba ser por tanto diferente en ambos grupos, intentando que la preservación de linfáticos sea mucho más efectiva en adolescentes debido a la alta probabilidad de desarrollo de hidrocele postquirúrgico $(28,29)$.

Con respecto al empleo de colorantes (azul de isosulfan o azul de metileno) para teñir los linfaticos y ayudar a su identificación para evitar seccionarlos durante la diseccion, todavía no se ha demostrado con estudios a largo plazo de casos/controles su fiabilidad y los posibles efectos secundarios de su inyección intraescrotal o intraparenquimatosa. Probablemente sean una opción de futuro pero la recomendación de su uso generalizado precisa de más evidencias científicas (30-32).

\section{CONCLUSIONES}

Según nuestro estudio, la corrección laparoscópica del varicocele es una técnica segura, eficaz e idónea para su empleo en pacientes pediátricos y adolescentes. La complicación más indeseable y frecuente es el hidrocele reactivo, que a veces puede aparecer mas de un año después de la intervención. Debido a este hecho, la incidencia de hidrocele descrita en los estudios publicados puede ser incluso inferior a la real.

\section{BIBLIOGRAFÍA y LECTURAS RECOMENDADAS (*lectura de interés $y^{* *}$ lectura fundamental)}

1. Diamond DA, Xuewu J, Cilento BG Jr, Bauer SB, Peters CA, Borer JG, et al. Varicocele surgery: a decade's experience at a children's hospital. BJU Int. 2009;104(2):246-9.

2. Greenfield SP, Seville P, Wan J. Experience with varicoceles in children and young adults. J Urol 2002;168:1684-8. 
3. Esposito C, Valla JS, Yeung CK. Current indications for laparoscopy and retroperitoneoscopy in pediatric urology. Surg Endosc 2004;18:1559-64.

4. Okuyama A, Nakamura M, Namiki M, Takeyama M, Utsunomiya M, Fujioka H, et al. Surgical repair of varicocele at puberty: preventive treatment for fertility improvement. J Urol 1988;139:562-5.

5. Agarwal A, Deepinder F, Cocuzza M, Agarwal R, Short RA, Sabanegh E, et al. Efficacy of varicocelectomy in improving semen parameters: new metaanalytical approach. Urology 2007;70: 532-8.

6. Palomo A. Radical cure of varicocele by a new technique: preliminary report. J Urol 1949;61:604-7.

7. Ivanissevich O. Left varicocele due to reflux: experience with 4470 operative cases in forty-two years. J Int Coll Surg 1960;34:742.

8. May M, Johannsen M, Beutner S, Helke C, Braun $\mathrm{KP}$, Lein M, et al. Laparoscopic surgery versus antegrade scrotal sclerotherapy: retrospective comparison of two different approaches for varicocele treatment. Eur Urol 2006;49:384-7.

9. Watanabe M, Nagai A, Kusumi N, Tsuboi H, Nasu Y, Kumon H. Minimal invasiveness and effectivity of subinguinal microscopic varicocelectomy: a comparative study with retroperitoneal high and laparoscopic approaches. Int J Urol 2005;12:892-8.

10. Beutner S, May M, Hoschke B, Helke C, Lein M, Roigas J, et al. Treatment of varicocele with reference to age: a retrospective comparison of three minimally invasive procedures. Surg Endosc 2007;21:61-5.

11. Sautter T, Sulser T, Suter S, Gretener H, Hauri D. Treatment of varicocele: a prospective randomized comparison of laparoscopy versus antegrade sclerotherapy. Eur Urol 2002; 41:398-400.

*12. Jimenez Garrido A, Garcia de la Torre MV, Sanchez de Badajoz E. A decade of laparoscopic varicocelectomy: costs and learning stages. Arch Esp Urol 1999;52:245-8.

**13. Misseri R, Gershbein AB, Horowitz M, Glassberg KI. The adolescent varicocele. II: the incidence of hydrocele and delayed recurrent varicocele after varicocelectomy in a long term follow-up. BJU Int 2001;87:494-8.

**14. Esposito C, Valla JS, Najmaldin A, Shier F, Mattioli $\mathrm{G}$, Savanelli A, et al. Incidence and management of hydrocele following varicocele surgery in children. J Urol 2004;171: 1271-3.

*15. Hassan JM, Adams MC, Pope $4^{\text {th }}$ JC, Demarco RT, Brock $3^{\text {rd }}$ JW. Hydrocele formation following laparoscopic varicocelectomy. J Urol 2006;175:1076-9.

16. Dubin L, Amelar RD. Varicocele size and results of varicocelectomy in selected subfertile men with varicocele. Fértil Steril 1970;21:606-9.

*17. Méndez-Gallart R, Bautista-Casasnovas A, EstevezMartínez E, Varela-Cives R. Laparoscopic Palomo varicocele surgery: lessons learned after 10 years' follow up of 156 consecutive pediatric patients. J Pediatr Urol. 2009;5(2):126-31.

18. Abdulmaaboud MR, Shokeir AA, Farage Y, Abd el-Rahman A, El-Rakhawy MM, Mutabagani H. Treatment of varicocele: a comparative study of conventional open surgery, percutaneous retrograde sclerotherapy, and laparoscopy. Urology 1998;52:294-300.

19. Vanderbrink BA, Palmer LS, Gitlin J, Levitt SB, Franco I. Lymphatic-sparing laparoscopic varicocelectomy versus microscopic varicocelectomy: is there a difference? Urology 2007;70: 1207-10.

**20. Glassberg KI, Poon SA, Gjertson CK, Decastro GJ, Misseri R. Laparoscopic lymphatic sparing varicocelectomy in adolescents. J Urol 2008;180:326-30.

21. Riccabona M, Oswald J, Koen M, Lusuardi L, Radmayr C, Bartsch G. Optimizing the operative treatment of boys with varicocele: sequential comparison of 4 techniques. J Urol 2003;169:666-8.

22. Koyle MA, Oottamasathien S, Barqawi A, Rajimwale A, Furness $3^{\text {rd }}$ PD. Laparoscopic Palomo varicocele ligation in children and adolescents: results of 103 cases. J Urol 2004; 172:1749-52.

23. McManus MC, Barqawi A, Meacham RB, Furness $3^{\text {rd }} \mathrm{PD}$, Koyle MA. Laparoscopic varicocele ligation: are there advantages compared with the microscopic subinguinal approach? Urology 2004;64:357-60.

24. Storm DW, Hogan MJ, Jayanthi VR. Initial experience with percutaneous selective embolization: A truly minimally invasive treatment of the adolescent varicocele with no risk of hydrocele development. J Pediatr Urol. 2010 Feb 9. [Epub ahead of print]

25. Lisle R, Mahomed A. Lymphatic sparing laparoscopic Palomo varicocelectomy. J Pediatr Surg 2010; 45: 285.

*26. Barroso U Jr, Andrade DM, Novaes H, Netto JM, Andrade J. Surgical treatment of varicocele in children with open and laparoscopic Palomo technique: a systematic review of the literature. J Urol. 2009; 181: 2724-8.

27. Healey C, Lisle R, Mahomed A. Outcome of Lymphatic- and Artery-Sparing Surgery for Pediatric Varicococele. J Laparoendosc Adv Surg Tech A. 2010 Mar 11. [Epub ahead of print]

28. Kocvara R, Dolezal J, Hampl R, Povysil C, Dvoracek J, Hill M. et al. Division of lymphatic vessels at varicocelectomy leads to testicular oedema and decline in testicular function according to the LH-RH analogue stimulation test. Eur Urol 2003; 43: 430

*29. Poon SA, Kozakowski KA, Decastro GJ, Gjertson CK, Glassberg KI. Adolescent varicocelectomy: Postoperative catch-up growth is not secondary to lymphatic ligation. J Pediatr Urol (2009) 5: 37-41

*30. Schwentner C, Radmayr C, Lunacek A, Gozzi C, Pinggera GM, Neururer R, et al. Laparoscopic varicocele ligation in children and adolescents using isosulphan blue: a prospective randomized trial. BJU Int 2006;98:861-5.

31. Oswald J, Korner I, Riccabona M. The use of isosulphan blue to identify lymphatic vessels in high retroperitoneal ligation of adolescent varicocele avoiding postoperative hydrocele. BJU Int 2001;87:502-4.

32. Rabah DM, Adwan AA, Seida MA. Lymphatic preservation using methylene blue dye during laparoscopic varicocelectomy: early results. Can J Urol. 2009; 16: 4826-30. 